\title{
Axial vibration confinement in nonhomogenous rods
}

\author{
S. Choura ${ }^{\mathrm{a}}$, S. EL-Borgi ${ }^{\mathrm{a}}$ and A.H. Nayfeh ${ }^{\mathrm{b}, *}$ \\ ${ }^{a}$ Applied Mechanics and Systems Research Laboratory, Tunisia Polytechnic School, B.P. 743, La Marsa 2078, \\ Tunisia \\ ${ }^{\mathrm{b}}$ Department of Engineering Science and Mechanics, MC 0219, Virginia Polytechnic and State University, \\ Blacksburg, VA 24061, USA
}

Received 16 January 2004

Revised 20 August 2004

\begin{abstract}
A design methodology for the vibration confinement of axial vibrations in nonhomogenous rods is proposed. This is achieved by a proper selection of a set of spatially dependent functions characterizing the rod material and geometric properties. Conditions for selecting such properties are established by constructing positive Lyapunov functions whose derivative with respect to the space variable is negative. It is shown that varying the shape of the rod alone is sufficient to confine the vibratory motion. In such a case, the vibration confinement requires that the eigenfunctions be exponentially decaying functions of space, where the notion of spatial domain stability is introduced as a concept dual to that of the time domain stability. It is also shown that vibration confinement can be produced if the rod density and/or stiffness are varied with respect to the space variable while the cross-section area is kept constant. Several case studies, supporting the developed conditions imposed on the spatially dependent functions for vibration confinement in vibrating rods, are discussed. Because variation in the geometric and material properties might decrease the critical buckling loads, we also discuss the buckling problem.
\end{abstract}

Keywords: Axial vibration, vibration confinement, material and geometric properties, buckling

\section{Introduction}

Flexible structures, such as aerospace and ship structures, large communication antennas, and seismically excited buildings and bridges, are exposed to vibration due to various external and/or internal excitations. They often excite unwanted structural resonances, which can cause damage or transmission of vibrational energy to distant parts or regions where they cannot be tolerated. Therefore, it may be of interest to remove the vibrational energy from the more sensitive parts of the structure and transfer it to the less sensitive parts. The sensitive parts of flexible structures are defined to be a set of spatial regions in which vibrations must be eliminated to ensure a better structural performance and less chances of destructing these structures. The sensitive parts include end-effectors of flexible robot manipulators.

Allaei investigated the feasibility of developing an efficient vibration-control methodology based on mode localization and termed it as vibration control by confinement [1]. The advantage of the confinement approach over conventional control in isolating the sensitive parts of a structure was demonstrated with several examples by Allaei [2]. The eigenvector assignment has been demonstrated to be an efficient tool for vibration control [3,19,22]. It is a means of redistributing the vibrational energy in the structure, and thus allowing the sensitive parts to converge to their steady-state values at fast rates.

\footnotetext{
*Corresponding author. E-mail: anayfeh@vt.edu.
} 
The eigenstructure assignment procedure has found application in a wide variety of control problems, such as those associated with flight-control design [13,21] and the control of vibrating structures [15]. An extensive literature is concerned with the development of algorithms for the computation of feedback gains that yield a desired set of closed-loop eigenvalues and eigenvectors [9-11,15,23].

Another design technique known as 'Inverse Eigenvalue Problem' arises in control-system design, system identification, structural analysis, mechanical system simulation, etc. . . The essential idea of an inverse eigenvalue problem is to reconstruct a matrix from prescribed spectral data, which may consist of the complete or only partial information of the eigenvalues or eigenvectors [7]. The objective of an inverse eigenvalue problem is to construct a matrix that maintains a specific structure and satisfies given spectral properties. Ram and Elhay have presented a method that constructs tridiagonal symmetric quadratic damping and stiffness matrices based on a given set of eigenvalues [17]. Sivan and Ram have treated the problem of determining structural alteration from a given set of eigenvalues and eigenvectors in the presence of model uncertainties [20]. Ram has synthesized control forces for shifting some poles of a vibrating rod to prescribed locations while keeping the other poles unchanged [16]. Ram and Elhay have reconstructed the shape of a rod with varying cross-section [18]. Their reconstruction is based on a discretized model of the rod leading to a specially structured matrix pencil.

Recently, Choura and Yigit have developed a strategy for the confinement of vibrations in flexible structures by distributed actuators [5]. The strategy consists of altering the original mode shapes of a flexible structure using feedback. The altered mode shapes are exponentially decaying functions of space that nearly vanish at the structural region where the vibrational energy is to be removed and transferred to the remaining parts. They have shown that it is possible to use an equivalent set of discrete actuators for confining the vibration [24]. The concept of using a reduced number of point actuators has been treated in [6]. As compared to the procedure of eigenstructure assignment offered by Shaw and Jayasuriya [19] and Song and Jayasuriya [22], Choura has developed a detailed assignment procedure for the purpose of vibration confinement [3]. Feedback forces have been used to allow parts of a structure reach their equilibria at fast rates at the expense of slowing down the convergence of the remaining parts to zero. The control strategy has been used to investigate the confinement and suppression of vibrations resulting from an initial energy distribution using feedback forces whose number is equal to that of the dimension of the discretized model. The strategy of vibration confinement by passive means has been applied to seismically excited structures [4].

In this paper, we consider the reconstruction (for existing structures) or design (for new structures) of rods with spatially varying shape and material properties by a proper selection of their cross-sectional areas, their modulii of elasticity, and/or their densities. The key idea of such a selection is to setup the ordinary-differential equation associated with the eigenfunctions as a space-domain stability problem dual to that of time-domain stability. Therefore, design techniques, such as pole placement and Lyapunov energy functions, can be used to determine the necessary conditions for assigning the spatially varying shape and material properties of the vibrating rod.

\section{Problem formulation}

Consider the axial vibration of a rod, shown in Fig. 1, with variable cross-sectional area $A(x)$, variable Young's modulus of elasticity, $E(x)$ and variable mass density per unit length $\rho(x)$, and governed by

$$
\partial x\left[E(x) A(x) \begin{array}{l}
\partial u \\
\partial x
\end{array}\right]-\rho(x) A(x) \begin{aligned}
& \partial^{2} u \\
& \partial t^{2}
\end{aligned}=0
$$

where the neutral axis is assumed to be linear.

It is known that the vibratory energy is equally distributed over the spatial domain of a rod having constant cross-section and constant material properties. One way to redistribute the energy in such a rod is to apply active control employing distributed force actuators [5] or point force actuators [24]. This paper proposes an alternate way for the redistribution of energy by modifying the rod's geometric and material properties. From a practical point of view, the designer is allowed to modify an already existing rod by adding and/or removing material. Therefore, the objective of this study is to determine a set of functions $A(x), E(x)$, and $\rho(x)$ such that the rod preserves a localization or confinement behavior during its vibrational motion in which certain parts of the rod will experience lower vibration amplitudes. 


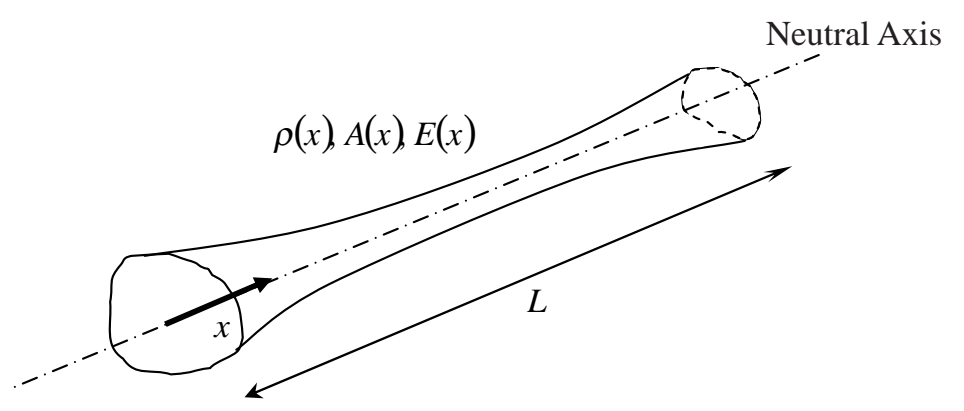

Fig. 1. Rod with variables cross section and variable material properties.

The axial displacement is expressed in the following form:

$$
u(x, t)=U(x) e^{j \omega t}
$$

where $U(x)$ is the eigenfunction, $\omega$ is the natural frequency of axial vibration, and $t$ is the time variable. Substituting Eq. (2) into Eq. (1) yields

$$
\begin{gathered}
d x \\
d x
\end{gathered}\left[\begin{array}{cc}
E(x) A(x) & d U(x) \\
d x
\end{array}\right]+\omega^{2} \rho(x) A(x) U(x)=0
$$

Vibration confinement can be defined as reducing the absolute value of $U(x)$ in the sensitive parts; that is,

$$
|U(x)|<\varepsilon \text { for } \forall x \in D_{1} \cup D_{2} \ldots \cup D_{n}
$$

where $\varepsilon$ is a small positive constant and the $D_{i}, i=1,2, \ldots, n$ correspond to the spatial intervals associated with the parts that are sensitive to vibration.

The strategy for vibration confinement proposed in this study converts the eigenfunctions of the original structure into exponentially decaying functions of the spatial coordinate $x$. If the size of the structure is large (i.e., the dimension of the rod's length $L$ is large), then spatial confinement of vibration becomes dual to time domain stability. It is, therefore, possible to design the functions $A(x), E(x)$, and $\rho(x)$ in Eq. (3) utilizing the classical pole placement techniques, such as using the Routh-Hurwitz criterion or the Lyapunov stability technique, and applying them to the spatial domain. In particular, the coefficients in Eq. (3) must be properly selected in order to "stabilize" the spatial dynamics, where the concept of spatial domain stability is introduced as a concept dual to that of the time-domain stability. Next, a strategy for selecting the above functions is developed for the general case followed by particular cases.

\section{Conditions for vibration confinement}

Consider the axial vibration of bars in which the cross-section area $A=A(x)$, Young's modulus of elasticity $E=E(x)$, and mass density per unit length $\rho=\rho(x)$ are all taken as variables. In this case, Eq. (3) can be rewritten as

$$
\left[p(x) U^{\prime}\right]^{\prime}+\omega_{p}^{2} \underset{(x)}{q(x)} U=0
$$

where $p(x)$ and $q(x)$ are positive functions for all $x$ and given by

$$
p(x)=E(x) A(x) \quad q(x)=\rho(x) E(x) A^{2}(x)
$$

In order to "stabilize" the spatial dynamics, a set of functions $A(x), E(x)$, and $\rho(x)$ can be found using the following candidate Lyapunov function:

$$
V(x)=U^{2}+\underset{\omega^{2} q(x)}{1}\left[p(x) U^{\prime}\right]^{2}>0
$$


Using Eq. (5), we simplify the derivative of $V(x)$ with respect to $x$ to

$$
V^{\prime}(x)=-\begin{gathered}
q^{\prime}(x) \\
\omega^{2} q(x)
\end{gathered}\left[p(x) U^{\prime}\right]^{2}
$$

which implies that

$$
q^{\prime}(x)=\left[\rho^{\prime}(x) E(x)+\rho(x) E^{\prime}(x)\right] A^{2}(x)+2 \rho(x) E(x) A(x) A^{\prime}(x)>0
$$

Equation (9) regulates the choice of the rod parameters and provides a relationship among them. It provides conditions on the choice of the physical and geometric parameters that lead to vibration confinement in the neighborhood of $x=0$. Conditions on these parameters for vibration confinement at an arbitrary point in the spatial domain are given later in this paper. From Eq. (9), it follows that the following cases are possible:

a. keep both of the cross-section area and the density constant across the rod and vary the modulus of elasticity provided that its spatial derivative with respect to $x$ is positive for all $x$. This implies that the rod stiffness increases and becomes larger in the neighborhood of the right end,

b. keep both of the cross-section area and the modulus of elasticity constant across the rod and vary the rod density provided that its spatial derivative with respect to $x$ is positive for all $x$. This implies that the rod density increases and becomes larger in the neighborhood of the right end,

c. keep the cross-section area constant across the rod and vary both of the modulus of elasticity and the density provided that the spatial derivative of their product with respect to $x$ is positive for all $x$. This implies that both of the rod stiffness and density increase simultaneously or one of them decreases and the other increases more rapidly. This is the case of a Functionally Graded Material (FGM), which is essentially a two-phase nonhomogeneous particulate composites synthesized in such a way that the volume fractions of the constituent materials, such as ceramic and metal, vary continuously along a spatial direction to give a predetermined composition profile, resulting in a relatively smooth variation of the mechanical properties [8].

Because variations in the geometric and material properties might decrease the critical buckling loads, we investigate the effect of the distributed geometric and physical properties on the buckling of a fixed-fixed rod. For a constant axial load, the static buckling problem associated with the rod is described by

$$
d x^{2}\left(E(x) I(x) \begin{array}{c}
d^{2} w \\
d x^{2}
\end{array}\right)+P_{d x^{2}}^{d^{2} w}=0 \quad w(0)=w(L)=0 ; \frac{d w}{d x}(0)=\frac{d w}{d x}(L)=0
$$

It follows from this boundary-value problem that only the modulus of elasticity and the second moment of area affect the buckling load $P$. The absence of the mass density shows that the cross-section area and the modulus of elasticity can be designed first to satisfy certain design conditions for the buckling problem. The mass density can then be designed to compensate for the confinement problem. This is demonstrated through a study case discussed later in this paper. Next, we give some examples of rods with spatially varying geometric and/or physical properties that demonstrate the validity of condition Eq. (9) and we also address the buckling problem for each example.

We first consider axial vibrations of a rod of length $L$ with variable cross-section area and constant physical properties; that is, $E(x)=E_{0}$ and $\rho(x)=\rho_{0}$. In this case, we search for possible forms of the cross-section that allow confinement of vibrations in preferred regions of the rod when the material properties are kept constant. In this case, Eq. (9) reduces to

$$
q^{\prime}(x)=2 \rho E A(x) A^{\prime}(x)>0
$$

which requires the cross-section area to be an increasing function of space over the domain $0<x<L$.

\section{An exponentially varying cross-section area}

In this case, the cross-section area is distributed according to the following exponential function:

$$
A(x)=A_{0} e^{\alpha x} \quad A(0)=A_{0} \text { and } A(L)=A_{L}
$$


where $\alpha={ }_{L}^{1} \ln \left(\begin{array}{l}A_{L} \\ A_{0}\end{array}\right)$. It follows from Eq. (12) that the area increases as the spatial variable $x$ increases. It can be shown that Eq. (3) simplifies to

$$
U^{\prime \prime}+\alpha U^{\prime}+{ }_{E_{0}}^{\rho_{0}} \omega^{2} U=0
$$

which in turn yields the characteristic equation

$$
\lambda^{2}+\alpha \lambda+{ }_{E_{0}}^{\rho_{0}} \omega^{2}=0
$$

Thus, the general solution is given by:

$$
\begin{aligned}
& U(x)=C_{1} e^{\lambda_{1} x}+C_{2} e^{\lambda_{2} x} \\
& \lambda_{1,2}=-{ }_{2}^{1} \alpha \pm \sqrt{4} \alpha^{2}-\begin{array}{c}
\rho_{0} \omega^{2} \\
E_{0}
\end{array}
\end{aligned}
$$

Next, we consider the cases of fixed-fixed and fixed-free rods, starting with the first.

For a fixed-fixed rod, the boundary conditions are given by $u(0, t)=0$ and $u(L, t)=0$, and the normalized eigenfunctions are given by

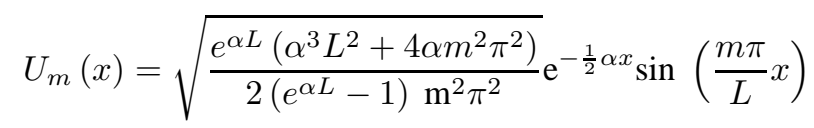

The scalar $\alpha$ must satisfy $0<\alpha<2 \sqrt{{ }_{E_{0}}} \omega_{1}$ and the natural frequencies are given by

$$
\omega_{m}^{2}=\begin{gathered}
E_{0} \\
\rho_{0}
\end{gathered}\left[\begin{array}{c}
m^{2} \pi^{2} \\
L^{2}
\end{array}+{ }_{4}^{1} \alpha^{2}\right], m=1,2,3, \ldots
$$

For a fixed-free rod, the boundary conditions are $u(0, t)=0$ and $u^{\prime}(L, t)=0$. The solution of equation (13) that satisfies the boundary condition $u(0, t)=0$ is given by

$$
U_{m}(x)=C_{m} \mathrm{e}^{-\frac{1}{2} \alpha x} \sin (\eta x)
$$

where

$$
\eta=\sqrt{\begin{array}{c}
\rho_{0} \omega_{m}^{2} \\
E_{0}
\end{array}-{ }_{4}^{1}} \alpha^{2}
$$

Imposing the boundary condition $u^{\prime}(L, t)=0$ yields the characteristic equation

$$
\cot (\eta L)=\begin{gathered}
\alpha \\
2 \eta
\end{gathered}
$$

Equation (21) does not have a closed-form solution and, therefore, either numerical or asymptotic methods are needed for approximating the natural frequencies. The large roots of the characteristic equation can be obtained using asymptotic methods as follows. When $\eta$ is large, the right-hand side of Eq. (21) can be neglected and hence we obtain $\cot (\eta L)=0$ whose solution is given by

$$
\eta L=\left(m+\begin{array}{l}
1 \\
2
\end{array}\right) \pi
$$

where $m$ is an integer. Then the solution of Eq. (21) can be sought in the form

$$
\eta L=\left(m+\begin{array}{l}
1 \\
2
\end{array}\right) \pi+\delta
$$

Substituting Eq. (23) into Eq. (21) yields

$$
\cot \left[\left(m+\begin{array}{l}
1 \\
2
\end{array}\right) \pi+\delta\right]=\begin{gathered}
\alpha L \\
(2 m+1) \pi+2 \delta
\end{gathered}
$$


Using trigonometric identities, we rewrite Eq. (24) as

$$
\begin{array}{cc}
\cot \left[\left(m+{ }_{2}^{1}\right) \pi\right]-\tan (\delta) \\
1+\tan (\delta) \cot \left[\left(m+{ }_{2}^{1}\right) \pi\right]
\end{array}=\begin{gathered}
\alpha L \\
(2 m+1) \pi+2 \delta
\end{gathered}
$$

or

$$
-\tan (\delta)=\begin{gathered}
\alpha L \\
(2 m+1) \pi+2 \delta
\end{gathered}
$$

For small $\delta$, Eq. (26) yields

$$
\delta=-\begin{gathered}
\alpha L \\
(2 m+1) \pi
\end{gathered}
$$

Therefore, to the second approximation,

$$
\eta L=\left(m+\frac{1}{2}\right) \pi-\left(\begin{array}{c}
\alpha L \\
(2 m+1) \pi
\end{array}\right.
$$

Substituting Eq. (28) into Eq. (20), we obtain, to the second approximation, the natural frequencies

$$
\begin{gathered}
\rho_{0} \omega_{m}^{2} \\
E_{0}
\end{gathered}=\frac{1}{2} \alpha^{2}+\frac{1}{L^{2}}\left[\left(m+\frac{1}{2}\right) \pi-\frac{\alpha L}{(2 m+1) \pi}\right]^{2}
$$

Equation (19) shows that the mode shapes are exponentially decaying functions of the spatial variable $x$. Higher values of $\alpha$ lead to faster decay of these eigenfunctions, and therefore a better confinement of the vibrational energy in the neighborhood of $x=0$. Figure 2 shows the first three modes and time response to an initial time velocity distribution with constant physical and geometric properties of a 10-meter aluminum $\operatorname{rod}\left(E=1.5 \times 10^{6} \mathrm{~N} / \mathrm{m}^{2}\right.$, $\rho=8760 \mathrm{~kg}$, and $A=0.01322 \mathrm{~m}^{2}$ ). Clearly, the vibratory energy is distributed throughout the rod at all times. Figures 3 and 4 show the shape of a square cross-section area, the first three eigenfunctions, and the time response to an initial time velocity distribution for fixed-fixed and fixed-free 10-meter aluminum rods $\left(E_{0}=1.5 \times 10^{6} \mathrm{~N} / \mathrm{m}^{2}\right.$, $\rho_{0}=8760 \mathrm{~kg}, A_{0}=10^{-4} \mathrm{~m}^{2}, A_{L}=9 \times 10^{-2} \mathrm{~m}^{2}$ and $\left.\alpha=0.68\right)$. As expected, the vibratory motion is confined in the left region of the rod. We note that the frequencies shown in figure 4 correspond to the exact values. The first three approximate frequencies obtained from Eq. (29) are, respectively, 6.8605, 10.6816, and 14.6745, which are close to the exact values.

Next, we address the buckling problem; the second moment of area is given by $I(x)=I_{0} e^{2 \alpha x}$. Hence, Eq. (10) becomes

$$
\frac{d^{2}}{d x^{2}}\left(\begin{array}{r}
\mathrm{e}^{2 \alpha x} d^{2} w \\
d x^{2}
\end{array}\right)+\kappa^{2}{ }^{d^{2} w} d x^{2}=0 \quad w(0)=w(L)=0 ; \frac{d w}{d x}(0)={ }_{d x}^{d w}(L)=0
$$

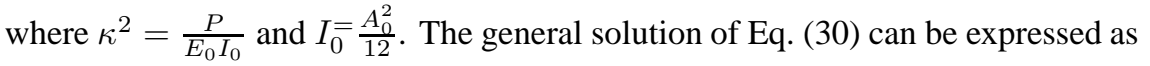

$$
w(x)=C_{1}+C_{2} x+C_{3} J_{0}\left(\begin{array}{l}
\kappa \\
\alpha
\end{array} \mathrm{e}^{-\alpha x}\right)+C_{4} Y_{0}\left(\begin{array}{l}
\kappa \\
\alpha
\end{array} \mathrm{e}^{-\alpha x}\right)
$$

where $J_{n}$ and $Y_{n}$ are the Bessel functions of the first and second kinds of order $n$, respectively. Applying the boundary conditions and using Mathematica, we find that the characteristic equation leads to the following lowest five critical axial loads: $P_{1}=20.5 E_{0} I_{0}, P_{2}=39.1 E_{0} I_{0}, P_{3}=77.8 E_{0} I_{0}, P_{4}=114.2 E_{0} I_{0}$, and $P_{5}=171.9 E_{0} I_{0}$.

For comparison purposes, we investigate the buckling problem associated with a 10-meter aluminum rod having constant physical and geometric properties $\left(E(x)=E_{0}\right.$ and $\left.I(x)=17466 I_{0}\right)$ where the second moment of area is calculated by assuming that the material volumes of both rods are the same. For the uniform beam, the buckling problem is given by

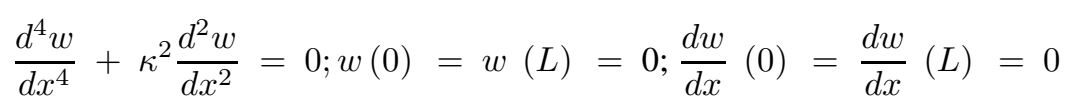

The general solution of Eq. (32) can be expressed as

$$
w(x)=C_{1}+C_{2} x+C_{3} \cos (\kappa x)+C_{4} \sin (\kappa x)
$$



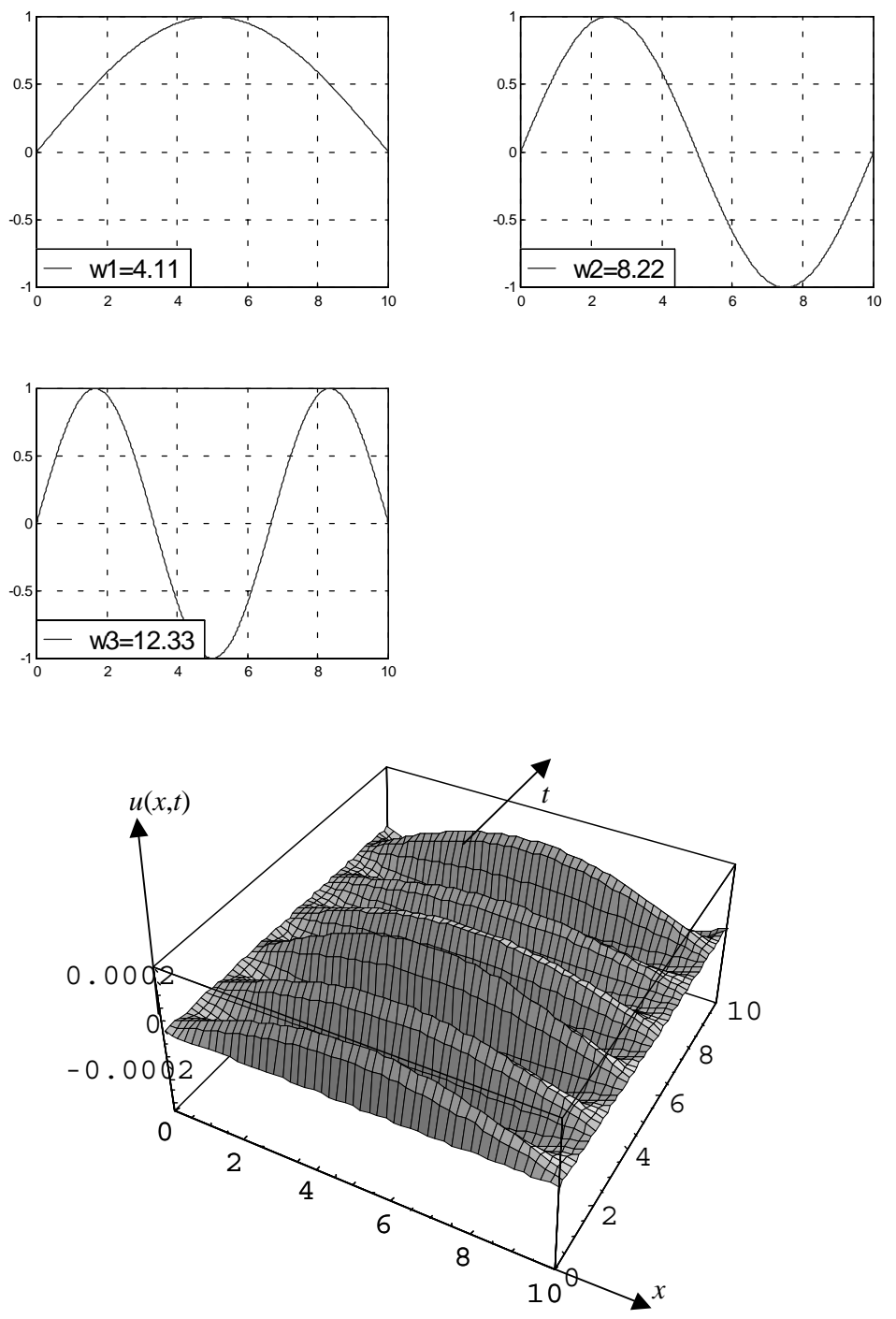

Fig. 2. First three eigenfunctions and time response of an aluminum fixed-fixed rod.

Applying the boundary conditions, we obtain the characteristic equation

$$
\kappa L \sin (\kappa L)+2 \cos (\kappa L)=2
$$

Using asymptotic methods, we find that the large roots of Eq. (34) are given by

$$
\kappa L=n \pi+\begin{gathered}
2[1-\cos (n \pi)] \\
n \pi \cos (n \pi)
\end{gathered} \quad \text { for } n \geqslant 2
$$

The lowest five exact and approximate roots are:
Exact roots:
$\kappa L=6.2832,8.9868,12.5664,15.4505$, and 18.8496
Approximate roots:
$\kappa L=6.2832,9.0004,12.5664,15.4533$, and 18.8496

We note that the roots in Eq. (35) corresponding to even values of $n$ are exact and those corresponding to odd values of $n$ are approximate. The lowest five critical buckling loads obtained numerically as well as from the asymptotic solution are:

$$
P_{1}=39.48 E_{0} I, P_{2}=80.65 E_{0} I, P_{3}=157.91 E_{0} I, P_{4}=238.68 E_{0} I \text {, and } P_{5}=355.31 E_{0} I .
$$



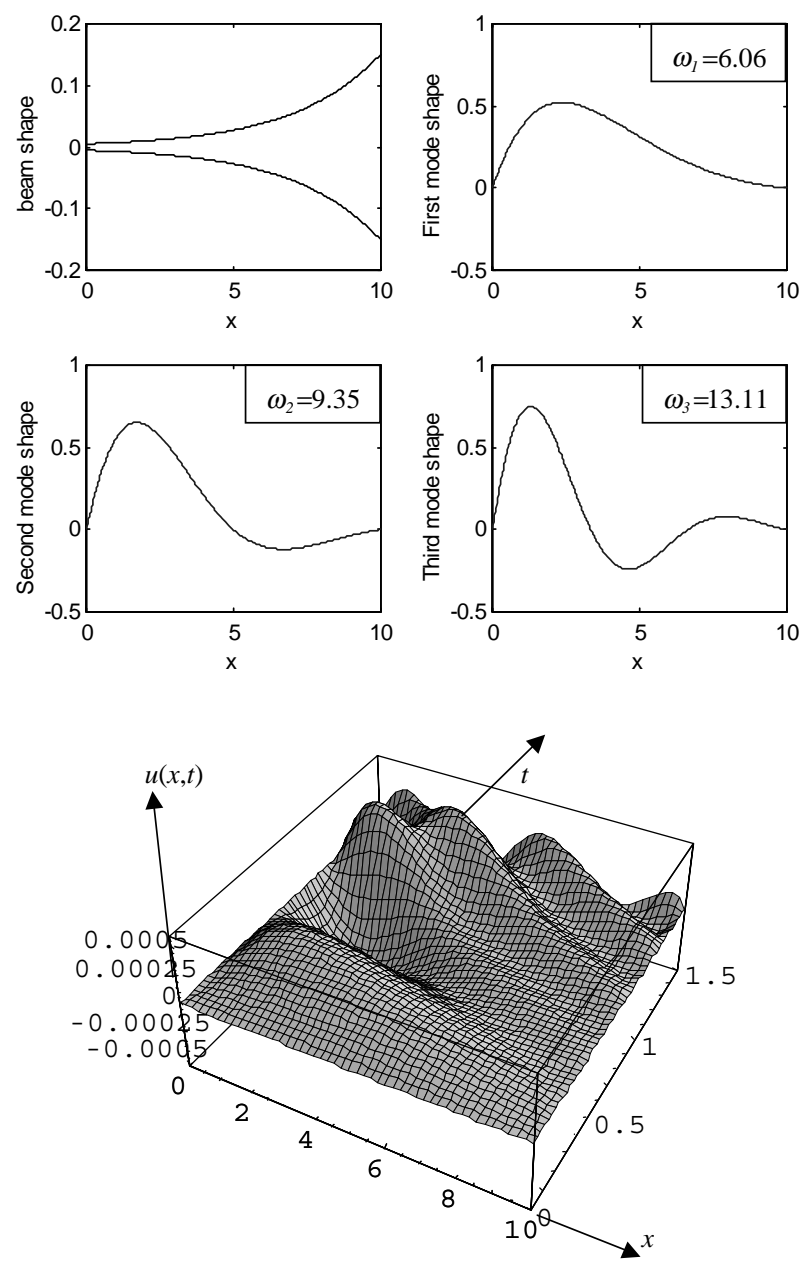

Fig. 3. Shape, first three eigenfunctions and time response of an aluminum fixed-fixed rod.

But $I=17466 I_{0}$, therefore the lowest five critical buckling loads are:

$$
P_{1}=689534 E_{0} I_{0}, P_{2}=1410612 E_{0} I_{0}, P_{3}=2758135 E_{0} I_{0}, P_{4}=4169471 E_{0} I_{0}, \text { and } P_{5}=6205803 E_{0} I_{0} .
$$

Comparison of the set of critical axial loads associated with a rod having spatially varying cross-section area with the above values reveals that the first critical value is decreased remarkably. This constitutes a disadvantage in the sense that improving vibration confinement results in a weak rod resistance to buckling in case the material volume is kept the same for both rods. This observation is valid only for rods having constant material properties and variable geometric properties. On the other hand, if the cross-section area is kept at the smallest value associated with the spatially varying rod $A_{0}$, then the first five critical axial loads become: $P_{1}=0.81 E_{0} I_{0}, P_{2}=2.39 E_{0} I_{0}$, $P_{3}=4.76 E_{0} I_{0}, P_{4}=7.91 E_{0} I_{0}$, and $P_{5}=11.86 E_{0} I_{0}$. In such a case, varying the cross-section area improves simultaneously the confinement of vibration and the rod resistance to buckling.

In order to avoid the decrease in the critical buckling loads, we investigate possibilities of varying the material properties with or without varying the geometric properties. This is discussed thoroughly in Sections 6-8.

\section{Linearly varying cross-section area}

For generally varying cross-section areas, 



Fig. 4. Shape, first three eigenfunctions and time response of an aluminum fixed-free rod.

$$
A(x)=A_{0} e^{\int_{0}^{x} f(\sigma) d \sigma}
$$

and Eq. (3) takes the form

$$
U^{\prime \prime}+f(x) U^{\prime}+{ }_{E_{0}}^{\rho_{0}} \omega^{2} U=0
$$

According to the spatial stability condition given in Eq. (11), the function $f(x)$ must be positive for all $x$. This condition implies that the cross-section is monotonically increasing function of $x$.

Next, we consider the case of linearly varying cross-section areas; that is,

$A(x)=A_{0}+{ }^{\left(A_{L}-A_{0}\right)} x$ corresponding to $f(x)=\begin{gathered}A_{L}-A_{0} \\ A_{0} L+\left(A_{L}-A_{0}\right)\end{gathered}$, where $A_{L}>A_{0}$ and $\alpha=\begin{gathered}A_{L}-A_{0} \\ L A_{0}\end{gathered}$.

In this case, the rod is stiffer in the vicinity of the right end. Then, the solution of Eq. (37) that vanishes at the left end is given by

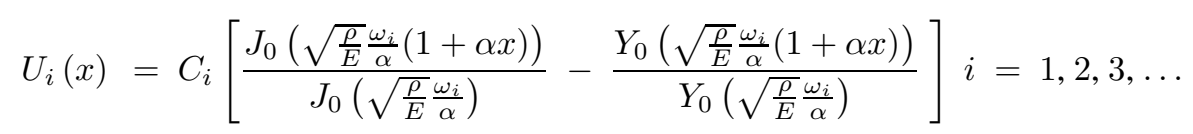

Then the corresponding characteristic equation is

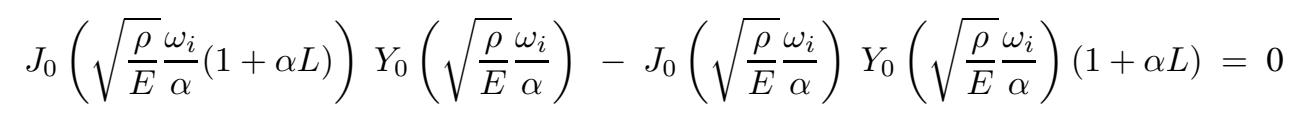



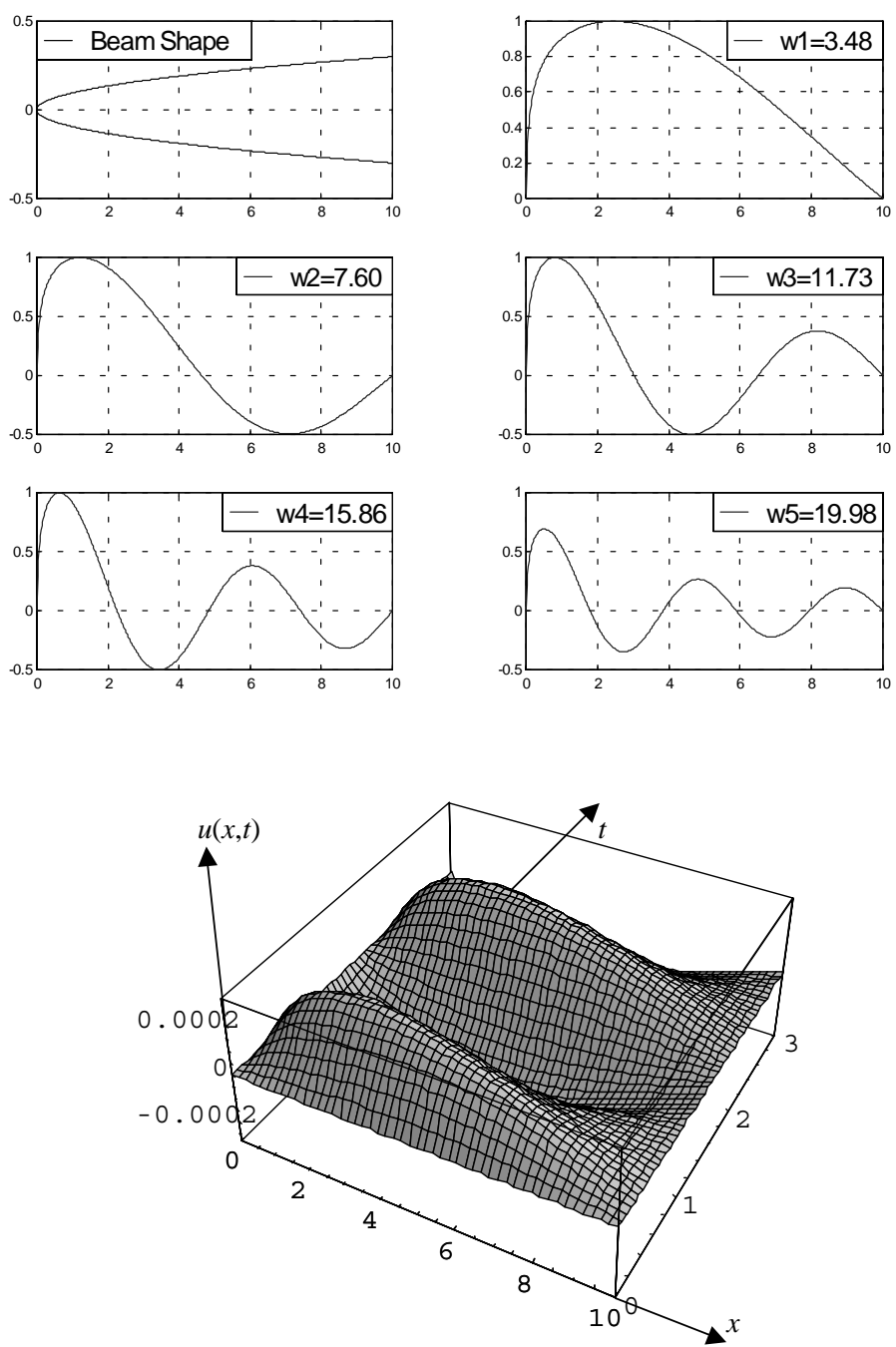

Fig. 5. Shape, first five eigenfunctions, and time response of an aluminum fixed-fixed rod with a linearly increasing area.

Figure 5 shows the rod shape and the first five eigenfunctions for $\left(E_{0}=1.510^{6} \mathrm{~N} / \mathrm{m}^{2}, \rho_{0}=8760 \mathrm{~kg}\right.$, $A_{0}=10^{-4} \mathrm{~m}^{2}$, and $\left.A_{L}=910^{-2} \mathrm{~m}^{2}\right)$. The time response of the aluminum rod for an initial time velocity distribution, shown in the same figure, depicts that the vibrations are confined towards the left end $(x=0)$.

In this case, the associated buckling problem is given by

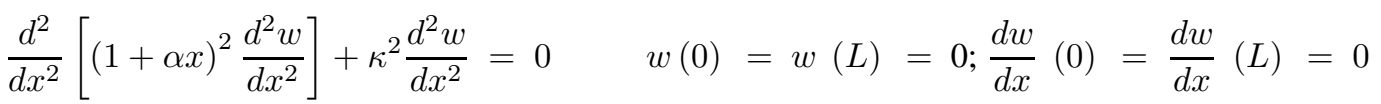

The general solution of Eq. (40) can be expressed as

$$
w(x)=C_{1}+C_{2} x+\sqrt{ } 1+\alpha x\left(C_{3} \cos \left[\begin{array}{c}
\sqrt{ } 4 \kappa^{2}-\alpha^{2} \ln (1+\alpha x) \\
2 \alpha
\end{array}\right]+C_{4} \sin \left[\begin{array}{c}
\sqrt{ } 4 \kappa^{2}-\alpha^{2} \ln (1+\alpha x) \\
2 \alpha
\end{array}\right]\right)
$$

Applying the boundary conditions to Eq. (41) yields the characteristic equation

$$
\begin{aligned}
& L\left(2 \kappa^{2}-\alpha^{2}\right) \sin \left[\begin{array}{c}
\sqrt{ } 4 \kappa^{2}-\alpha^{2} \ln (1+\alpha L) \\
2 \alpha
\end{array}\right]+(2+\alpha L) \sqrt{ } 4 \kappa^{2}-\alpha^{2} \cos \left[\begin{array}{c}
\sqrt{ } 4 \kappa^{2}-\alpha^{2} \ln (1+\alpha L) \\
2 \alpha
\end{array}\right] \\
& =2 \sqrt{ }(1+\alpha L)\left(4 \kappa^{2}-\alpha^{2}\right)
\end{aligned}
$$



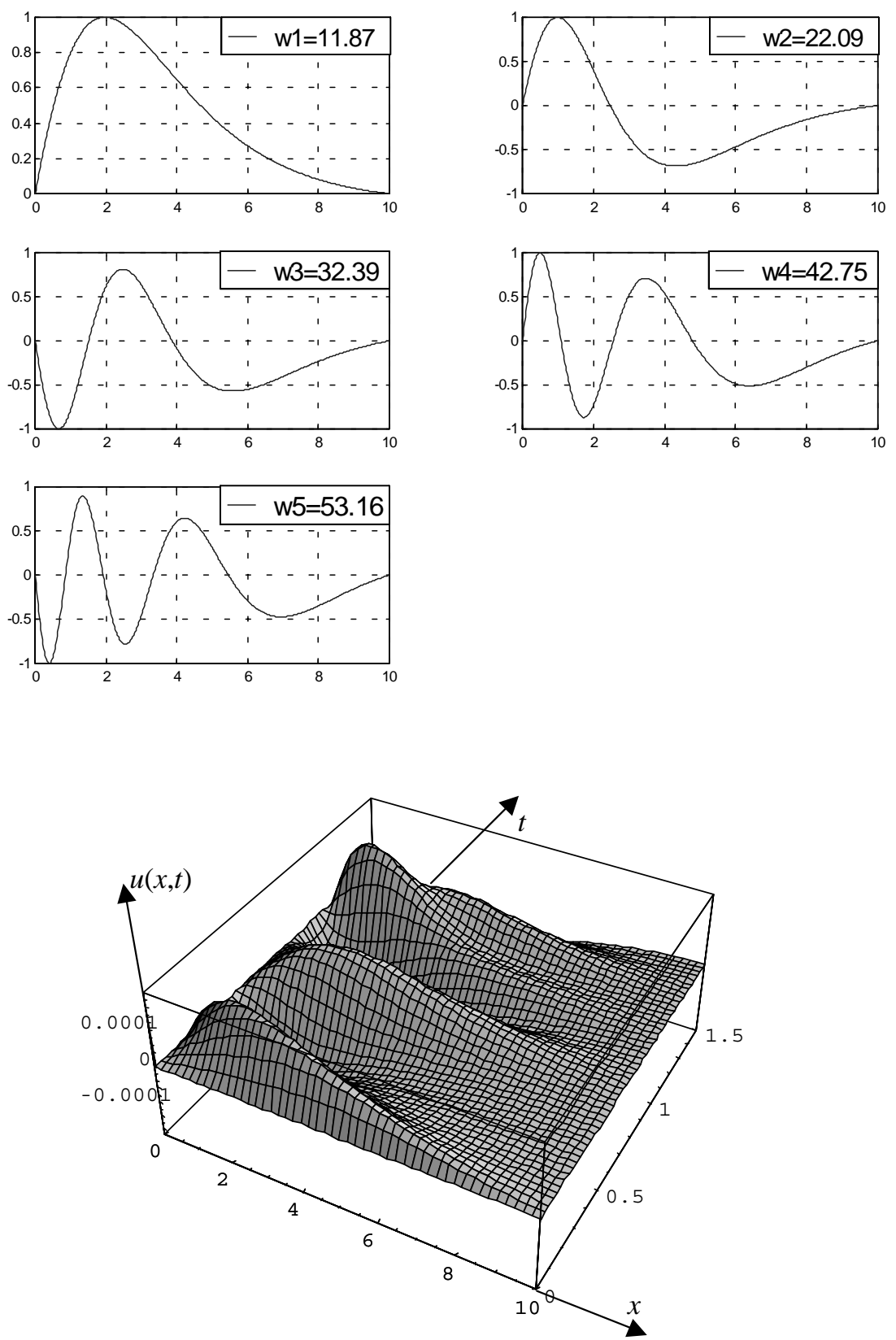

Fig. 6. First five eigenfunctions and time response of an aluminum fixed-free rod with an exponentially increasing modulus of elasticity.

The large roots of Eq. (42) are given by

$$
\begin{gathered}
P \\
E_{0} I_{0}
\end{gathered}={ }_{4}^{1} \alpha^{2}+\begin{gathered}
4 n^{2} \pi^{2} \alpha^{2} \\
\ln ^{2}(1+\alpha L)
\end{gathered}-\begin{gathered}
8 n^{2} \pi^{2} \alpha[2+\alpha L-2 \sqrt{ } 1+\alpha L \cos (n \pi)] \\
L \ln (1+\alpha L)\left[4 n^{2} \pi^{2}-\ln ^{2}(1+\alpha L)\right]
\end{gathered}
$$

The characteristic Eq. (42) leads to the following first five critical axial loads: $P_{1}=6710 E_{0} I_{0}, P_{2}=15020 E_{0} I_{0}$, $P_{3}=27382 E_{0} I_{0}, P_{4}=42585 E_{0} I_{0}$, and $P_{5}=61857 E_{0} I_{0}$.

For comparison purposes, we investigate the buckling problem associated with a 10-meter aluminum rod with constant physical and geometric properties $\left(E(x)=E_{0}\right.$ and $\left.I(x)=202950.25 I_{0}\right)$. The second moment of area is found on the basis that the material volume of both rods is the same. The associated characteristic equation 
leads to the following first five critical axial loads: $P_{1}=80122 E_{0} I_{0}, P_{2}=163909 E_{0} I_{0}, P_{3}=320486 E_{0} I_{0}$, $P_{4}=484479 E_{0} I_{0}$, and $P_{5}=965230 E_{0} I_{0}$. Comparison of the two sets of critical axial loads shows that the relative differences are reduced remarkably in this case. The buckling problems in Sections 4 and 5 reveal that the spatial variation of the cross-section area plays a major role in controlling the relative differences in the critical buckling loads.

\section{Exponentially varying modulus of elasticity}

In this case, $A(x)=A_{0}, \rho(x)=\rho_{0}, E(x)=E_{0} \mathrm{e}^{\alpha x}$ with $\alpha={ }_{L}^{1} \ln E_{L} / E_{0}$ and $E_{L}>E_{0}$; that is, the rigidity increases exponentially with respect to $x$ making the rod stiffer in the vicinity of the right end. In practice, this could be the case of an FGM with constituent materials having the same density but different moduli of elasticity. Thus, Eq. (3) reduces to

$$
d x\left[\begin{array}{c}
\mathrm{e}^{\alpha x} d U \\
d x
\end{array}\right]+\omega^{2}{ }_{E_{0}}^{\rho_{0}} U=0
$$

For a fixed-fixed rod, the eigenfunctions are given by

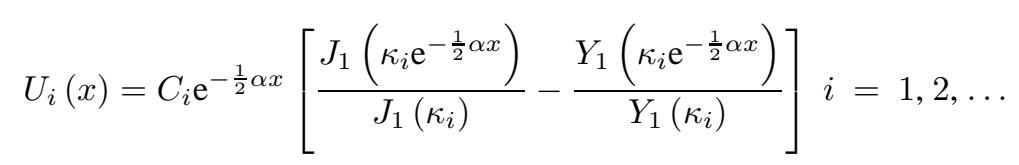

where $\kappa_{i}^{2}=\begin{gathered}4 \rho_{0} \omega_{i}^{2} \\ \alpha^{2} E_{0}\end{gathered}$. The corresponding characteristic equation is

$$
J_{1}\left(\kappa_{i} \mathrm{e}^{-\frac{1}{2} \alpha L}\right) Y_{1}\left(\kappa_{i}\right)-J_{1}\left(\kappa_{i}\right) Y_{1}\left(\kappa_{i} \mathrm{e}^{-\frac{1}{2} \alpha L}\right)=0
$$

For a 10 -meter aluminum $\operatorname{rod}\left(E_{0}=1.510^{6} \mathrm{~N} / \mathrm{m}^{2}, E_{L}=1.510^{8} \mathrm{~N} / \mathrm{m}^{2}, \rho_{0}=8760 \mathrm{~kg}, L=10 \mathrm{~m}\right.$, and $A_{0}=10^{-4} \mathrm{~m}^{2}$ ), Fig. 6 shows the first five eigenfunctions and the time response for an initial time velocity distribution. This simulation confirms the confinement of vibrations in the neighborhood of the left end $(x=0)$.

As in previous cases, the associated buckling problem is defined as

$$
d x^{2}\left[\begin{array}{r}
\mathrm{e}^{\alpha x} d^{2} w \\
d x^{2}
\end{array}\right]+\begin{gathered}
P d^{2} w \\
E_{0} I_{0} d x^{2}
\end{gathered}=0 \quad w(0)=w(L)={ }_{d x}^{d w}(0)=\begin{gathered}
d w \\
d x
\end{gathered}(L)=0
$$

It can be verified that the solution associated with the above boundary-value problem can be expressed as

$$
w(x)=C_{1}+C_{2} x+C_{3} J_{0}\left(\lambda \mathrm{e}^{-\frac{1}{2} \alpha x}\right)+C_{4} Y_{0}\left(\lambda \mathrm{e}^{-\frac{1}{2} \alpha x}\right)
$$

where $\lambda^{2}=\begin{gathered}4 P \\ \alpha^{2} E_{0} I_{0}\end{gathered}$. Applying the boundary conditions and letting $L=10 \mathrm{~m}$, we obtain from the characteristic equation the following first five critical axial buckling loads: $P_{1}=2.68 E_{0} I_{0}, P_{2}=5.30 E_{0} I_{0}, P_{3}=10.47 E_{0} I_{0}$, $P_{4}=15.65 E_{0} I_{0}$, and $P_{5}=23.40 E_{0} I_{0}$.

For comparison purposes, we investigate the buckling problem associated with a 10-meter aluminum rod with constant physical and geometric properties $\left(E(x)=E_{0}\right.$ and $\left.I(x)=I_{0}\right)$. The second moment of area is kept the same since the material volume of both rods is kept the same. The first five critical axial loads are found to be $P_{1}=0.81 E_{0} I_{0}, P_{2}=2.39 E_{0} I_{0}, P_{3}=4.76 E_{0} I_{0}, P_{4}=7.91 E_{0} I_{0}$, and $P_{5}=11.86 E_{0} I_{0}$. In this case, the first critical axial load is enhanced, and therefore, a proper spatial variation of the modulus of elasticity produces simultaneous improvement in the vibration confinement and resistance to buckling.

We note that the homogeneous rod uses the minimum value of the spatially varying modulus of elasticity $E(x)=E_{0} \mathrm{e}^{\alpha x}$. In case the average value ${ }_{2}^{1}\left(E_{0}+E_{L}\right)=50.05 E_{0}$ is employed, then the critical buckling loads become $P_{1}=40.54 E_{0} I_{0}, P_{2}=119.62 E_{0} I_{0}, P_{3}=238.24 E_{0} I_{0}, P_{4}=395.90 E_{0} I_{0}$, and $P_{5}=593.59 E_{0} I_{0}$. In this case, the critical loads associated with the spatially varying rod are lower than those of a homogeneous rod. 


\section{Exponentially varying density}

In this case, $A(x)=A_{0}, E(x)=E_{0}, \rho(x)=\rho_{0} \mathrm{e}^{\beta x}$ with $\beta={ }_{L}^{1} \ln { }^{\rho_{L}}$ and $\rho_{L}>\rho_{0}$; that is, the density increases exponentially with respect to $x$ making the rod more dense in the vicinity of the right end. In practice, this could be the case of an FGM with constituent materials having the same modulus of elasticity but different densities. Thus, Eq. (3) reduces to

$$
\frac{d^{2} U}{d x^{2}}+{ }_{E_{0}}^{\rho_{0} \omega^{2}} \mathrm{e}^{\beta x} U=0
$$

For a fixed-fixed rod with, the eigenfunctions satisfying Eq. (49) are given by

$$
U_{i}(x)=C_{i}\left[\begin{array}{cc}
J_{0}\left(\kappa_{i} \mathrm{e}^{\frac{1}{2} \beta x}\right) & Y_{0}\left(\kappa_{i} \mathrm{e}^{\frac{1}{2} \beta x}\right) \\
J_{0}\left(\kappa_{i}\right) & Y_{0}\left(\kappa_{i}\right)
\end{array}\right] i=1,2,3, \ldots
$$

where $\kappa_{i}^{2}={ }_{\beta^{2} E_{0}}^{4 \rho_{0} \omega_{i}^{2}}$. The corresponding characteristic equation is

$$
J_{0}\left(\kappa_{i} \mathrm{e}^{\frac{1}{2} \beta L}\right) Y_{0}\left(\kappa_{i}\right)-J_{0}\left(\kappa_{i}\right) Y_{0}\left(\kappa_{i} \mathrm{e}^{\frac{1}{2} \beta L}\right)=0
$$

Figure 7 shows the first five eigenfunctions and the time response for an initial time velocity distribution for a rod with the properties: $E_{0}=1.510^{6} \mathrm{~N} / \mathrm{m}^{2}, \rho_{0}=8760 \mathrm{~kg}, \rho_{L}=87600 \mathrm{~kg}, L=10 \mathrm{~m}$, and $A_{0}=10^{-4} \mathrm{~m}^{2}$. This figure clearly shows that the vibrations are confined towards the left end $(x=0)$.

\section{Exponentially varying density and modulus of elasticity}

In this case, $A(x)=A_{0}, \rho(x)=\rho_{0} \mathrm{e}^{\beta x}$ and $E(x)=E_{0} \mathrm{e}^{\alpha x}$; that is, all physical properties increase exponentially with respect to $x$ making the rod stiffer and denser in the vicinity of the right end. In practice, this could be the case of an FGM with constituent materials having different densities and different moduli of elasticity. Thus, Eq. (3) reduces to

$$
d x\left[\mathrm{e}^{\alpha x} d U\right]+{ }_{E_{0}}^{\rho_{0} \omega^{2}} \mathrm{e}^{\beta x} U=0
$$

For a fixed-fixed rod, the eigenfunctions satisfying Eq. (52) are given by

$$
U_{i}(x)=C_{i} \mathrm{e}^{-\frac{1}{2} \alpha x}\left[\begin{array}{cc}
J_{\nu}\left(\kappa_{i} \mathrm{e}^{-\frac{1}{2}(\alpha-\beta) x}\right) & Y_{\nu}\left(\kappa_{i} \mathrm{e}^{-\frac{1}{2}(\alpha-\beta) x}\right) \\
J_{\nu}\left(\kappa_{i}\right) & -J_{\nu}\left(\kappa_{i}\right)
\end{array}\right] i=1,2,3, \ldots
$$

where $\nu=\underset{\alpha-\beta}{\alpha}, \kappa_{i}^{2}=\underset{(\alpha-\beta)^{2} E_{0}}{4 \rho_{0} \omega^{2}}$, and $\alpha \neq \beta$. The corresponding characteristic equation is

$$
J_{\nu}\left(\kappa_{i} \mathrm{e}^{-\frac{1}{2}(\alpha-\beta) L}\right) Y_{\nu}\left(\kappa_{i}\right)-J_{\nu}\left(\kappa_{i}\right) Y_{\nu}\left(\kappa_{i} \mathrm{e}^{-\frac{1}{2}(\alpha-\beta) L}\right)=0
$$

When $\alpha=\beta$, the eigenfunctions that satisfy the left boundary condition can be expressed as

$$
U_{i}(x)=C_{i} \mathrm{e}^{-\frac{1}{2} \alpha x} \sin \left[\sqrt{ } \rho_{0} \omega_{i}^{2} / E_{0}-\alpha^{2} x\right] \quad i=1,2,3, \ldots
$$

Therefore, the natural frequencies are given by

$$
\omega_{i}=\sqrt{E_{0}} \rho_{0}\left(\begin{array}{c}
n^{2} \pi^{2} \\
L^{2}
\end{array}+\alpha^{2}\right) i=1,2,3, \ldots
$$

Figure 8 shows the first five eigenfunctions and time response for an initial time velocity distribution for a rod with the properties: $E_{0}=1.5 \times 10^{6} \mathrm{~N} / \mathrm{m}^{2}, \rho_{0}=8760 \mathrm{~kg}$, and $A_{0}=10^{-4} \mathrm{~m}^{2}, \alpha=\beta=\ln (10) / 10$. As it can be seen, the vibrations are confined in the vicinity of the left end $(x=0)$. The associated buckling problem is defined by Eq. (47) whose solution is given by Eq. (48). 

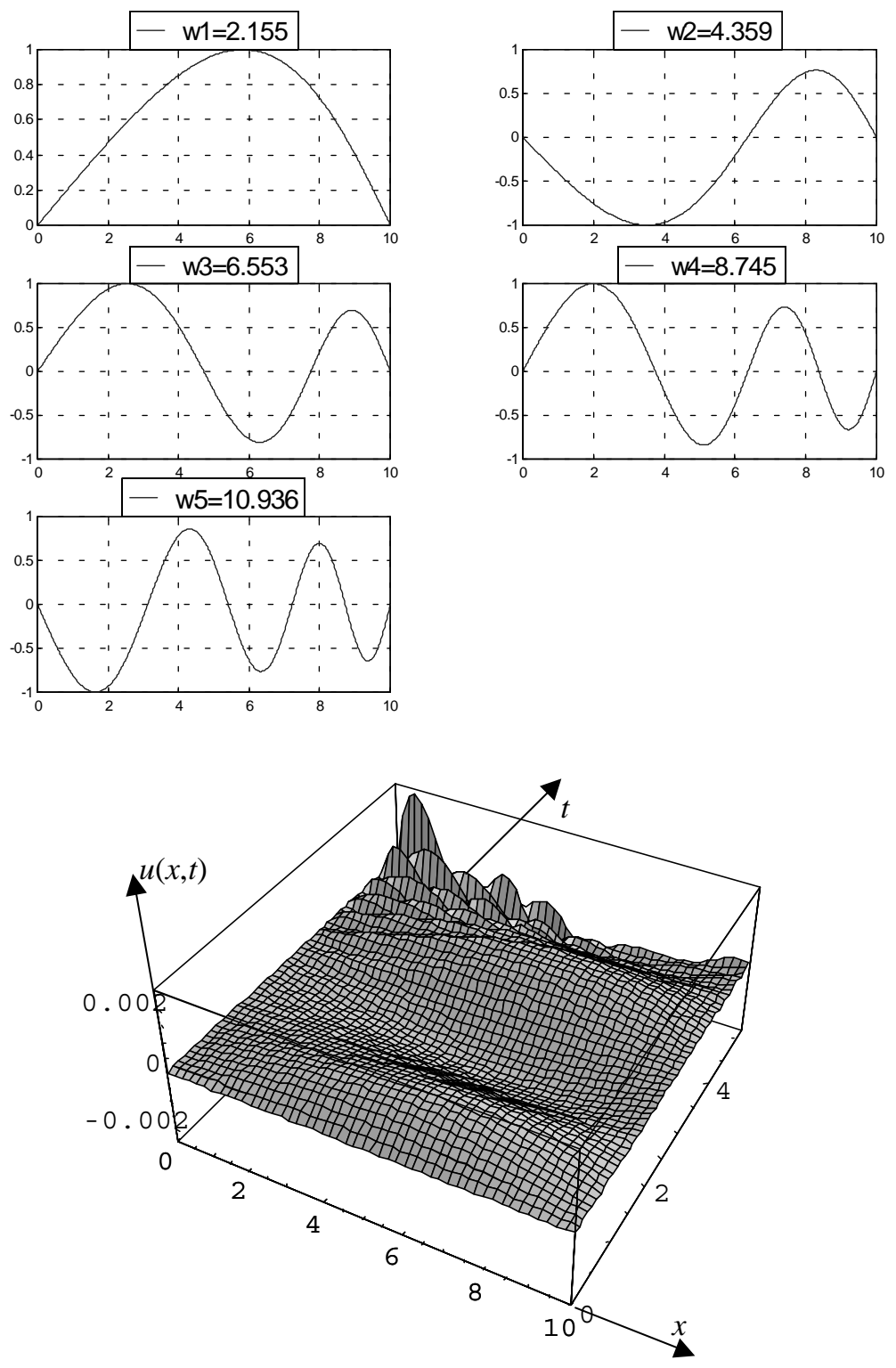

Fig. 7. First five eigenfunctions and time response of an aluminum fixed-fixed rod with an exponentially increasing density.

\section{Exponentially varying density, modulus of elasticity, and cross-section area}

This case presents an example of a rod in which all geometric and physical parameters vary with the spatial variable $x$ as $A(x)=A_{0} \mathrm{e}^{\gamma x}, \rho(x)=\rho_{0} \mathrm{e}^{{ }^{\prime} x}, E(x)=E_{0} \mathrm{e}^{\alpha} x$; that is, all material and geometric properties increase exponentially with $x$ making the rod stiffer, thicker, and denser in the vicinity of the right end. Then, the solution to this case is the same as in the preceding case with $\alpha=\alpha^{\prime}+\gamma$ and $\beta=\beta^{\prime}+\gamma$. Figure 9 shows the first five eigenfunctions and time response for an initial time velocity distribution for a rod with the properties: $E_{0}=1.510^{6} \mathrm{~N} / \mathrm{m}^{2}, \rho_{0}=8760 \mathrm{~kg}, A_{0}=10^{-4} \mathrm{~m}^{2}$, and $\alpha^{\prime}=\beta^{\prime}=\gamma=(\ln 10) / 10$. As it can be seen, the vibrations are confined in the vicinity of the left end $(x=0)$.

In this case, the solution to the buckling problem is similar to that in Section 8 where $2 \alpha=\alpha^{\prime}+2 \gamma$. The first five critical loads are: $P_{1}=9.41 E_{0} I_{0}, P_{2}=18.01 E_{0} I_{0}, P_{3}=35.86 E_{0} I_{0}, P_{4}=52.74 E_{0} I_{0}$, and $P_{5}=79.29 E_{0} I_{0}$. As compared to the preceding case, the critical loads are enhanced, and therefore, a proper spatial variation of all 

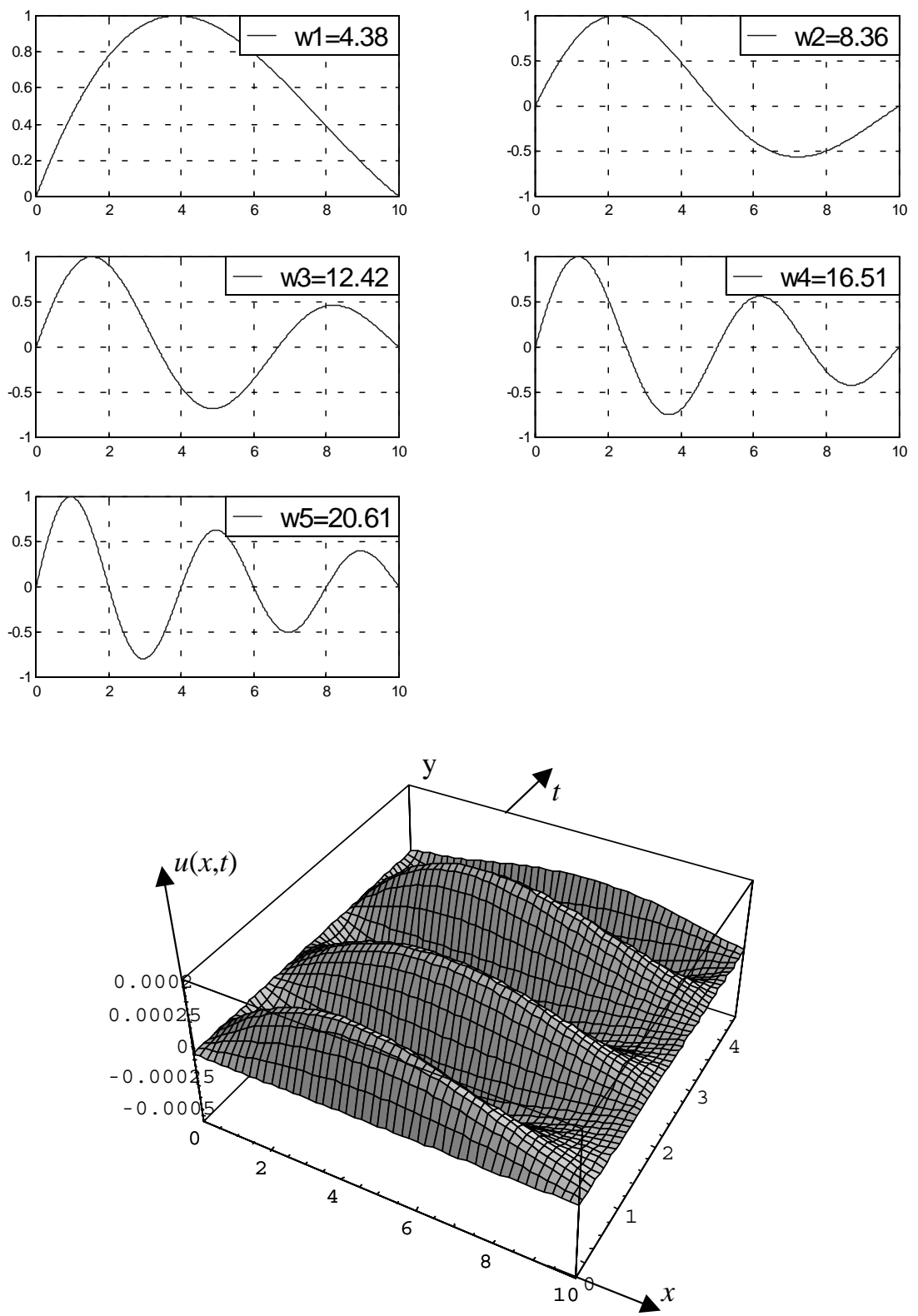

Fig. 8. First five eigenfunctions and time response of an aluminum fixed-fixed rod with an exponentially increasing density and modulus of elasticity.

parameters is capable of producing a simultaneous improvement of vibration confinement and rod resistance to buckling.

\section{Confinement at an interior region}

If one wishes to confine the vibrations about an arbitrary point $x_{0}$ instead of the origin, then the feedback force must be altered as follows: first, we shift the origin to $x_{0}$, the point about which the vibrations to be confined. Now, the boundary-value problems associated with the rod are:

$$
\left[p(x) U^{\prime}\right]^{\prime}+\omega_{p}^{2} \underset{p(x)}{q(x)} U=0 \quad-x_{0}<x<0
$$



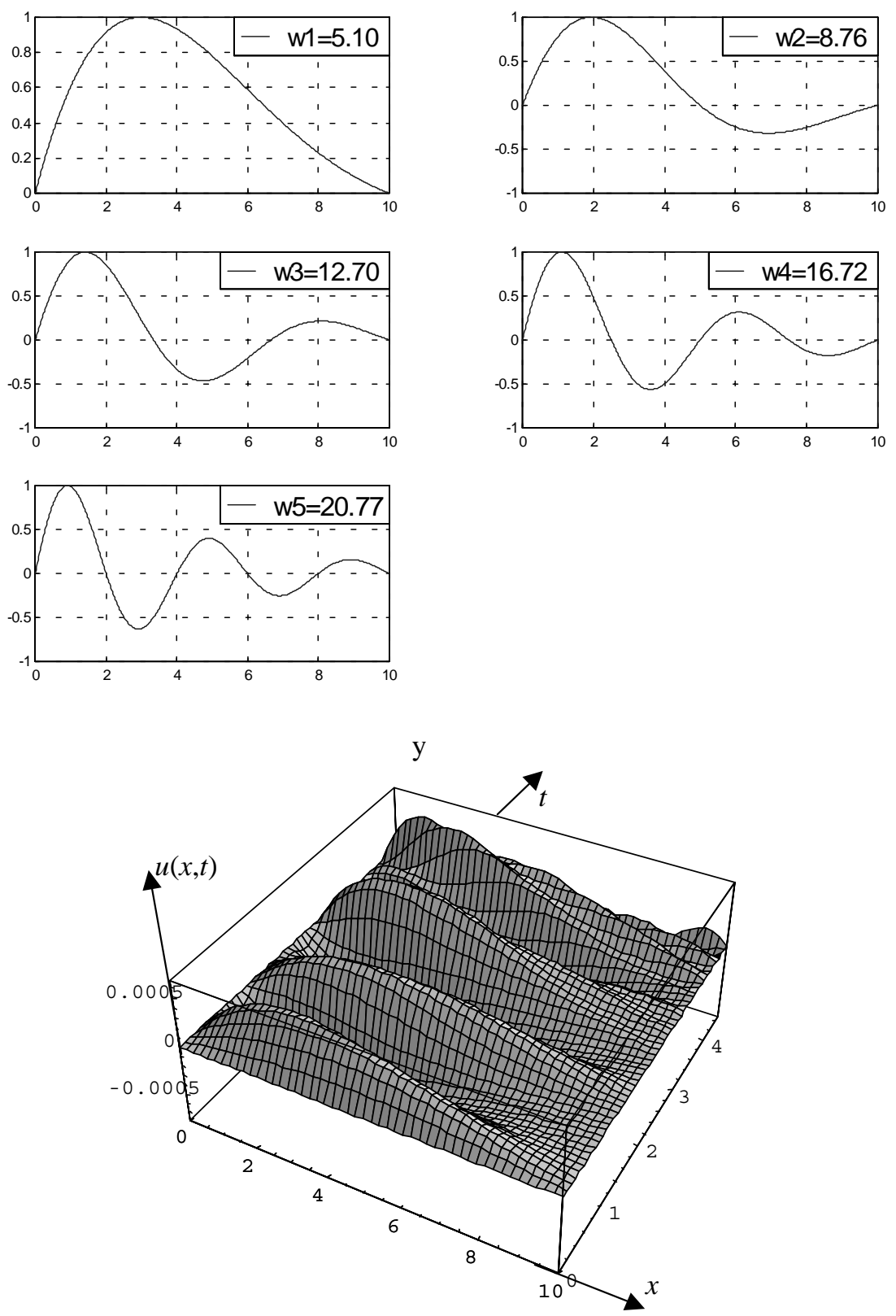

Fig. 9. First five eigenfunctions and time response of an aluminum fixed-fixed rod with an exponentially increasing cross-sectional area, density and modulus of elasticity.

$$
\left[p(x) U^{\prime}\right]^{\prime}+\omega_{p}^{2} \underset{p(x)}{q(x)} U=0 \quad 0<x<L-x_{0}
$$

The Lyapunov function and its space derivative given in Eqs (7) and (8) can be employed for the above boundaryvalue problems, but different conditions are obtained. Equation (9) becomes

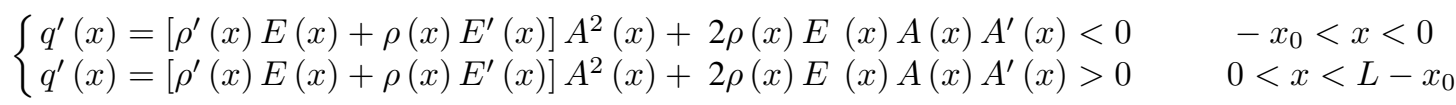

For particular cases, the above two conditions can be interpreted physically as follows: 


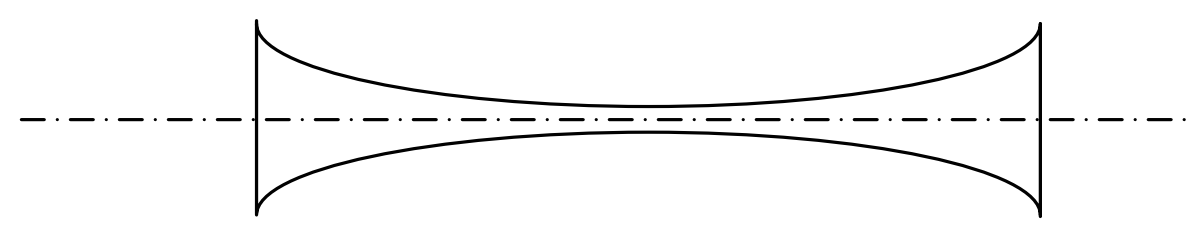

Fig. 10. Shape of a rod producing vibration confinement at the middle point.

1. keep both of the modulus of elasticity and the density constant across the rod and vary the cross-section area provided that its spatial derivative with respect to $x$ is negative for $-x_{0}<x<0$ and positive for $0<x<L-x_{0}$. This implies that the rod shape decreases up to $x_{0}$ and then increases up to the right end of the rod,

2. keep both of the modulus of elasticity and the cross-section area constant across the rod and vary the density provided that its spatial derivative with respect to $x$ is negative for $-x_{0}<x<0$ and positive for $0<x<L-x_{0}$. This implies that the rod density decreases up to $x_{0}$ and then increases up to the right end of the rod,

3. keep both of the density and cross-section area constant across the rod and vary the modulus of elasticity provided that its spatial derivative with respect to $x$ is negative for $-x_{0}<x<0$ and positive for $0<x<L-x_{0}$. This implies that the rod stiffness decreases up to $x_{0}$ and then increases up to the right end of the rod.

Figure 10 shows a particular shape of the rod that produces vibration confinement at the middle of the rod (both modulus of elasticity and density are constant and the cross-section area is variable). As another illustration, let us consider a beam whose modulus of elasticity varies according to the following distribution function:

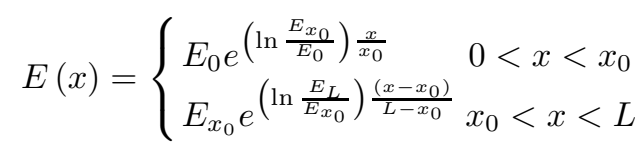

where $E_{0}>E_{x_{0}}$ and $E_{L}>E_{x_{0}}$. This implies that the modulus of elasticity is an exponentially decaying function of $x$ for $0<x<x_{0}$ and is an exponentially increasing function of $x$ in the interval $x_{0}<x<L$. For a rod of constant density $\left(\rho_{0}=8760 \mathrm{Kg} / \mathrm{m}^{3}\right)$, constant cross-section area $\left(A_{0}=10^{-4} \mathrm{~m}^{2}\right), L=10$ meter, $x_{0}=5$ meter, $E_{0}=E_{L}=1.5 \times 10^{8} \mathrm{~N} / \mathrm{m}^{2}$, and $E_{x_{0}}=1.5 \times 10^{6} \mathrm{~N} / \mathrm{m}^{2}$, Fig. 11 shows the first five mode shapes associated with the distribution given by Eq. (60). Applying the boundary conditions associated with the buckling problem, the characteristic equation leads to the following first five critical axial loads: $P_{1}=0.54 E_{0} I_{0}$, $P_{2}=3.91 E_{0} I_{0}, P_{3}=8.58 E_{0} I_{0}, P_{4}=14.31 E_{0} I_{0}$, and $P_{5}=16.27 E_{0} I_{0}$. Compared to the case of constant material and geometric properties, we conclude that the first critical buckling load is reduced by $33 \%$ while the second, third, fourth, and fifth critical loads are increased by $64 \%, 80 \%, 81 \%$ and $37 \%$, respectively.

\section{Conclusions}

This paper is concerned with the design of spatially-dependent functions characterizing structure material and geometric properties for the confinement of rod axial vibrations. The aim is to develop a set of rod parameters that lead to reallocating its vibratory energy so that vibrations are reduced in the sensitive parts and increased in the remaining parts. The selection of such properties is accomplished by constructing positive Lyapunov functions whose derivative with respect to the space variable is negative. In case both of the density and modulus of elasticity are kept constant while the cross-section area is varied, vibration confinement requires that the eigenfunctions be exponentially decaying functions of space, where the notion of spatial domain stability is introduced as a concept dual to that of time-domain stability. Thus, varying the shape of the rod alone is sufficient to confine the vibratory motion. We have also shown that vibration confinement can be produced if one (or both) of the rod density and stiffness is varied with respect to the space variable while the cross-section area is kept constant. Several cases, supporting the developed conditions imposed on the spatially-dependent functions, are discussed and simulated. Because variations 

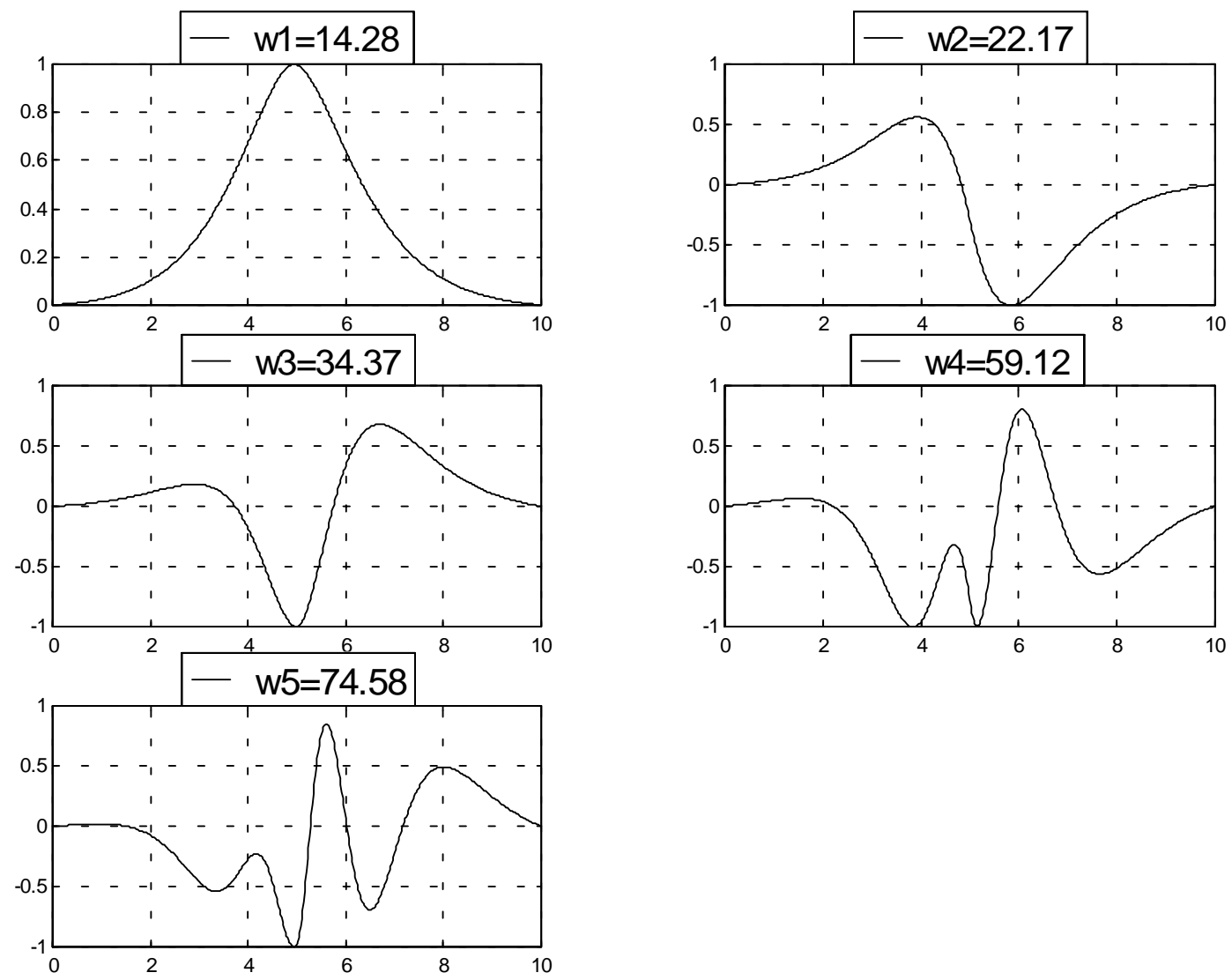

Fig. 11. First five eigenfunctions of an aluminum fixed-fixed rod with an exponentially decreasing-increasing modulus of elasticity.

in the geometric and physical properties of rods might affect their buckling, we have also addressed the buckling problem.

We believe that the outcome of this study for the axial vibration of rods can be extended for the vibratory motion of general 1-D and 2-D flexible structures, such as the flexural vibrations of beams and plates. This constitutes the focal point of the authors' future research in the area of passive control of flexible structures by confinement of vibrations.

\section{Acknowledgement}

This work was supported by the National Science Foundation under Grant No. INT-0138083.

\section{References}

[1] D. Allaei, Application of localized modes in vibration control, Proceedings of the 2nd International Congress on Recent Development of Air and Structure Borne Sound and Vibration, Auburn, Alabama, 1992.

[2] D. Allaei, Performance comparison between vibration control by confinement and conventional control techniques, Proceedings of the ASME 16th Biennial Conference on Mechanical Vibration and Noise, Sacramento, California, 1997.

[3] S. Choura, Control of flexible structures with the confinement of vibrations, ASME Journal of Dynamic Systems, Measurement, and Control 117(2) (1995), 155-164.

[4] S. Choura, S. El-Borgi, A. Abichou and A.H. Nayfeh, Control of seismically excited structures by passive confinement of vibrations, The 3rd World Conference on Structural Control, April 7-11, Como, Italy, 2002. 
[5] S. Choura and A.S. Yigit, Vibration confinement in flexible structures by distributed feedback, Journal of Computers and Structures 54(3) (1995), 531-540.

[6] S. Choura and A.S. Yigit, Confinement and suppression of structural vibrations, ASME Journal of Vibration and Acoustics 123 (October, 2001), 496-501.

[7] M.T.C. Chu, Inverse eigenvalue problems, SIAM Review 40 (1998), 1-39.

[8] F. Erdogan, Fracture Mechanics of Functionally Graded Materials, Composites Engineering 5 (1995), 753-770.

[9] M.M. Fahmy and J. O'Reilly, On eigenstructure assignment in linear multivariable system, IEEE Transactions on Automatic Control AC-27(3) (1982), 690-693.

[10] J.N. Juang, K.B. Lim and J.L. Junkins, Robust eigensystem assignment for flexible structures, Journal of Guidance, Control, and Dynamic 12 (1989), 311-317.

[11] J. Kautsy, N.K. Nichols and O. Van Dooren, Robust pole assignment in linear state Feedback, International Journal of Control 41(5) (1985), 1229-1245.

[12] T. Kobori and S. Kamagata, Dynamic intelligent buildings-active seismic response control, Intelligent Structures-2, 1992, pp. 279-282, Y.K. Wen, ed., Elsevier Applied Science, New York.

[13] B.S. Liebst and W.L. Garrard, Application of eigenspace techniques to design of aircraft control systems, Proceedings of the 1985 American Control Conference, June 1985, pp. 475-480, Boston, Massachusetts.

[14] P.G. Maghami and S. Gupta, On the eigensystem assignment with dissipativity constraints, Proceedings of the 1993 American Control Conference, June 1993, pp. 1271-1275, San Francisco, California.

[15] P.G. Maghami, J. Juang and K.B. Lim, Eigensystem assignment with output feedback, Journal of Guidance, Control, and Dynamics $\mathbf{1 5}$ (1993), 531-536.

[16] Y.M. Ram, Pole assignment for the vibrating rod, Quarterly Journal of Mechanics and Applied Mathematics 51(3) (1998), 477-492.

[17] Y.M. Ram and S. Elhay, An inverse eigenvalue problem for the symmetric tridiagonal quadratic pencil with application of damped oscillatory systems, SIAM Journal on Applied Mathematics 56(1) (1996), 232-244.

[18] Y.M. Ram and S. Elhay, Constructing the shape of a rod from eigenvalues, Communications in Numerical Methods in Engineering 14(7) (1998), 597-608.

[19] J. Shaw and S. Jayasuriya, Arbitrary assignment of eigenvectors with state feedback, ASME Journal of Dynamic Systems, Measurement and Control 114 (1992), 721-723.

[20] D.D. Sivan and Y.M. Ram, Physical modifications to vibratory systems with assigned eigendata, ASME Journal of Applied Mechanics 66(2) (1999), 427-432.

[21] K.M. Sobel, E.Y. Shapiro and A.N. Andry Jr., Eigenstructure assignment, International Journal of Control 59 (1994), $13-37$.

[22] B.K. Song and S. Jayasuriya, Active vibration control using eigenvector assignment for mode localization, Proceedings of the 1993 American Control Conference, June 1993, 1020-1024, San Francisco, California.

[23] R.F. Wilson, J.R. Cloutier and R.K. Yedaveli, Control design for robust eigenstructure assignment in linear uncertain systems, IEEE Control Systems Magazine 12(5) (1992), 29-34.

[24] A.S. Yigit and S. Choura, Vibration confinement in flexible structures via alteration of mode shapes using feedback, Journal of Sound and Vibration 179 (1995), 553-567. 

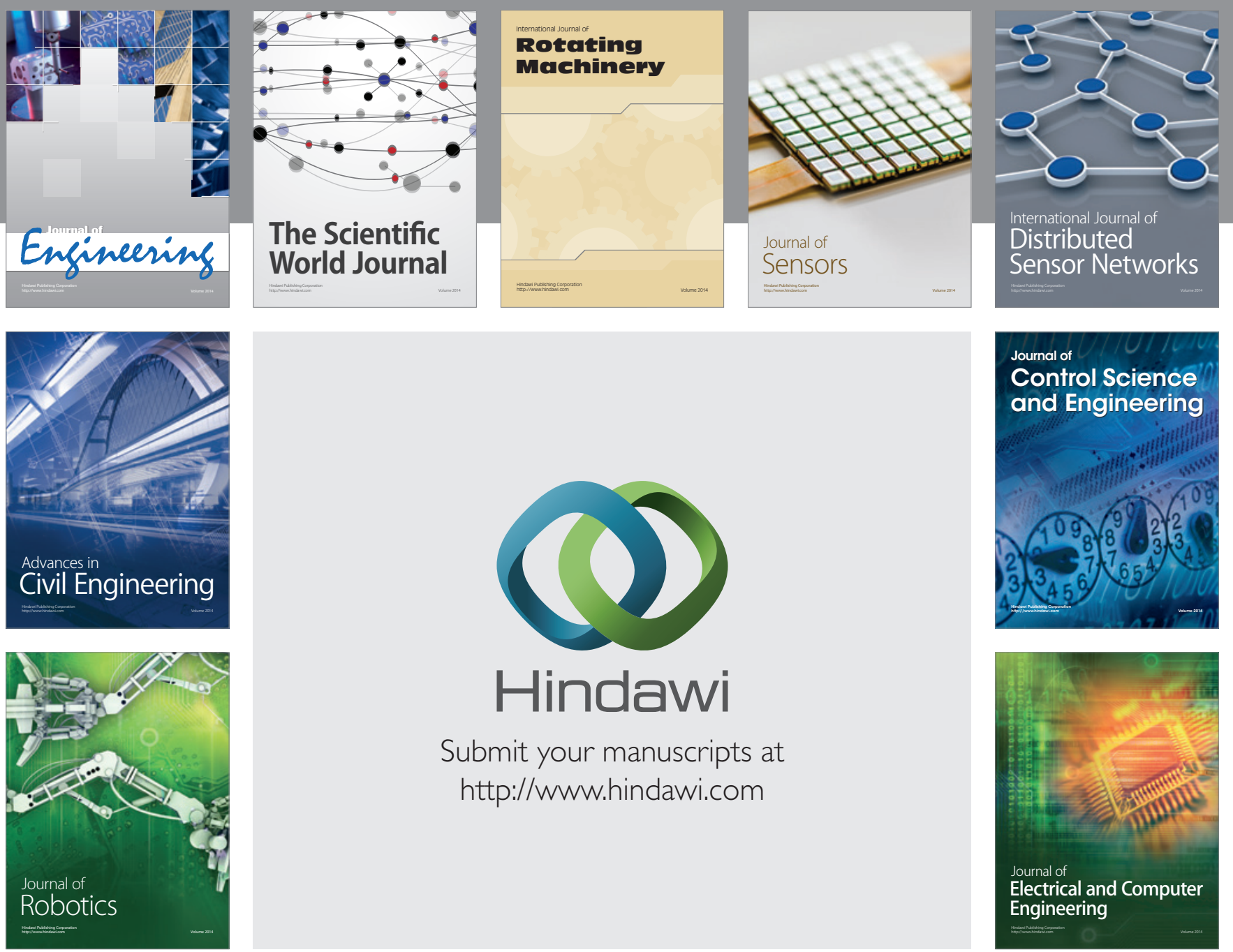

Submit your manuscripts at

http://www.hindawi.com
